\title{
POWER SUMS OF ROOTS OF A NONLINEAR SYSTEM
}

\author{
A. KYTMANOV, S. MYSLIVETS, AND N. TARKHANOV
}

\begin{abstract}
For a system of meromorphic functions $f=\left(f_{1}, \ldots, f_{n}\right)$ in $\mathbb{C}^{n}$, an explicit formula is given for evaluating negative power sums of the roots of the nonlinear system $f(z)=0$.
\end{abstract}

\section{Contents}

1. Introduction 1

2. Separating cycles 2

3. A residue integral 3

4. Transformation under an involution 4

5. Power sums 6

6. Generalisation to meromorphic maps $\quad 7$

7. Explicit evaluation 9

References 12

\section{INTRODUCTION}

An important tool of computer algebra and its applications in gas dynamics and chemical physics is the elimination of variables from a systems of nonlinear algebraic equations. The classical elimination method has been considerably modified on the base of residue theory. The logarithmic and Grothendieck residues are crucial ingredients of the new tools, cf. [AYu83], [BKL98], [Tsi92], etc.

The new approach consists of evaluating the sums of values of an arbitrary polynomial over all roots of the system, without having granted these roots. This allows one to find the power sums of roots and the resultant of the system relative to an arbitrary polynomial by using the classical Newton recurrence relations or their multidimensional analogues, cf. [BKL98].

For a nonlinear system $f(z)=0$ in $\mathbb{C}^{n}$ given by entire or meromorphic functions, the set of roots may be countable (if discrete). Hence, the power sums of roots fail in general to be well defined for positive powers. The aim of this paper is to study the power sums of roots for negative powers and derive an explicit evaluation formula for them.

Date: October 25, 2004.

2000 Mathematics Subject Classification. Primary 32A60; Secondary 13Pxx, 32A27.

Key words and phrases. Nonlinear systems, zeta-function, residue. 


\section{Separating CyCles}

Consider a system of holomorphic functions $f_{1}, \ldots, f_{n}$ in a neighbourhood of $0 \in \mathbb{C}^{n}$, each $f_{j}$ being of the form

$$
f_{j}(z)=z^{m_{j}} P_{j}(z)+Q_{j}(z)
$$

where $m_{j}=\left(m_{j, 1}, \ldots, m_{j, n}\right)$ is a multi-index with nonnegative integer entries, $P_{j}$ a homogeneous polynomial of degree $k_{j}$, and $Q_{j}$ a Taylor series in a neighbourhood of the origin.

We shall make two standing assumptions on the functions $P_{j}$ and $Q_{j}$ under consideration. Namely, the system $P_{1}, \ldots, P_{n}$ is required to have a unique common zero in $\mathbb{C}^{n}$, i.e., $z=0$. The assumption on $Q_{j}$ is that the degree of each monomial entering into $Q_{j}$ is strictly greater than the degree of $z^{m_{j}} P_{j}$, i.e., $\left|m_{j}\right|+k_{j}$ where $\left|m_{j}\right|:=m_{j, 1}+\ldots+m_{j, n}$.

For a fixed $\varepsilon=\left(\varepsilon_{1}, \ldots, \varepsilon_{n}\right)$ with $\varepsilon_{j}>0$, we introduce the cycle

$$
\Gamma_{P}=\left\{z \in \mathbb{C}^{n}:\left|P_{j}(z)\right|=\varepsilon_{j} \text { for } j=1, \ldots, n\right\}
$$

in $\mathbb{C}^{n}$.

Lemma 2.1. For almost all positive $\varepsilon_{1}, \ldots, \varepsilon_{n}$, the cycle $\Gamma_{P}$ is compact and smooth.

Proof. The smoothness of $\Gamma_{P}$ for almost all $\varepsilon$ follows from the Sard theorem. It remains to show that $\Gamma_{P}$ is compact.

Choose natural numbers $q_{1}, \ldots, q_{n}$, such that $q_{1} k_{1}=\ldots=q_{n} k_{n}=N$. For $R>0$, consider the set $B_{R}=\left\{z \in \mathbb{C}^{n}: \sum_{j=1}^{n}\left|P_{j}(z)\right|^{2 q_{j}} \leq R^{2}\right\}$. If we prove that $B_{R}$ is compact, we shall have established the compactness of $\Gamma_{P}$, for $\Gamma_{P} \subset B_{R}$ holds for sufficiently large $R$.

Pick $z \in B_{R}$. Write $z=t w$, where $t \in \mathbb{C}$ and $|w|=1$. Then

$$
\sum_{j=1}^{n}\left|P_{j}(z)\right|^{2 q_{j}}=|t|^{2 N} \sum_{j=1}^{n}\left|P_{j}(w)\right|^{2 q_{j}}
$$

Since the polynomials $P_{1}(w), \ldots, P_{n}(w)$ have no common zero on the unit sphere, it follows that

$$
\sum_{j=1}^{n}\left|P_{j}(w)\right|^{2 q_{j}} \geq c^{2}
$$

for $|w|=1$, with $c>0$ a constant independent of $w$. Therefore,

$$
\begin{aligned}
R^{2} & \geq|t|^{2 N} \sum_{j=1}^{n}\left|P_{j}(w)\right|^{2 q_{j}} \\
& \geq c^{2}|t|^{2 N},
\end{aligned}
$$

i.e., $|t|^{2 N} \leq \frac{R^{2}}{c^{2}}$ whence $|z| \leq \sqrt[N]{\frac{R}{c}}$, as desired.

Lemma 2.2. Suppose, for every $j=1, \ldots, n$, that either one of $P_{1}, \ldots, P_{n}$ vanishes identically on the hyperplane $z_{j}=0$ or there is a subsystem of $n-1$ polynomials which have the only common zero at the origin on $z_{j}=0$. Then there exist $\varepsilon_{1}, \ldots, \varepsilon_{n}>0$ such that $\Gamma_{P} \subset(\mathbb{C} \backslash\{0\})^{n}$. 
Proof. Consider the coordinate hyperplane $z_{j}=0$. If one of the polynomials $P_{1}, \ldots, P_{n}$ vanishes identically on $z_{j}=0$, then the cycle $\Gamma_{P}$ does not meet this hyperplane, as is easy to see.

We now assume that the system

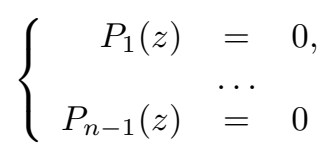

has a unique root on the hyperplane $z_{j}=0$, i.e., that at the origin. By Lemma 2.1 , the cycle $\Gamma_{P^{\prime}}$ on $z_{j}=0$, defined by $P^{\prime}=\left(P_{1}, \ldots, P_{n-1}\right)$, is compact. For any neighbourhood $U$ of 0 on $z_{j}=0$ one can choose $\varepsilon_{1}, \ldots, \varepsilon_{n-1}$ small enough, such that $\Gamma_{P^{\prime}} \subset U$. The set

$$
U=\left\{z \in \mathbb{C}^{n}: z_{j}=0 \text { and }\left|P_{n}(z)\right|<\varepsilon_{n}\right\}
$$

is open and contains 0 . It follows that $\Gamma_{P^{\prime}} \subset U$, hence $\Gamma_{P}$ does not intersect the hyperplane $z_{j}=0$.

In the sequel we will assume that $\varepsilon$ is chosen in such a way that $\Gamma_{P} \subset(\mathbb{C} \backslash\{0\})^{n}$ is fulfilled.

\section{A RESIDUE INTEGRAL}

Given any $s \in \mathbb{Z}^{n}$, we consider the integral

$$
\begin{aligned}
\mathcal{I}(s+I) & =\frac{1}{(2 \pi \imath)^{n}} \int_{\Gamma_{P}} z^{-s-I} \frac{d f}{f} \\
& =\frac{1}{(2 \pi \imath)^{n}} \int_{\Gamma_{P}} \frac{1}{z_{1}^{s_{1}+1} \cdot \ldots \cdot z_{n}^{s_{n}+1}} \frac{d f_{1}}{f_{1}} \wedge \ldots \wedge \frac{d f_{n}}{f_{n}} .
\end{aligned}
$$

Let us show that the integral is well defined, i.e., $f_{j} \neq 0$ on $\Gamma_{P}$ if $\varepsilon_{j}$ is small enough, for $j=1, \ldots, n$.

We get

$$
f_{j}(t z)=t^{\left|m_{j}\right|+k_{j}}\left(z^{m_{j}} P_{j}(z)+t F_{j}(t, z)\right)
$$

for any $t \in \mathbb{C}$. On the cycle $\Gamma_{P}$ each expression $z^{m_{j}} P_{j}(z)$ is bounded away from zero because

$$
\begin{aligned}
\left|z^{m_{j}} P_{j}(z)\right| & =\left|z^{m_{j}}\right| \varepsilon_{j} \\
& \geq c_{j}
\end{aligned}
$$

with $c_{j}>0$ a constant independent of $z$. Furthermore, the functions $F_{j}$ are bounded on the compact set $\{t \in \mathbb{C}:|t| \leq 1\} \times \Gamma_{P}$ in $\mathbb{C}^{n+1}$. We can thus assert that $\left|z^{m_{j}} P_{j}(z)\right|>\left|t F_{j}(t, z)\right|$ on $\Gamma_{P}$ for all $t$ small enough. Hence $\left|z^{m_{j}} P_{j}(z)\right|>\left|Q_{j}(z)\right|$ holds on any cycle

$$
t \Gamma_{P}=\left\{w \in \mathbb{C}^{n}:\left|P_{1}(w)\right|=|t|^{k_{1}} \varepsilon_{1}, \ldots,\left|P_{n}(w)\right|=|t|^{k_{n}} \varepsilon_{n}\right\}
$$

with sufficiently small $|t|$. It follows that $f_{j}(z) \neq 0$ on $t \Gamma_{P}$ for all $j=1, \ldots, n$, as desired.

Note that the integral $\mathcal{I}(s+I)$ is neither a logarithmic residue nor a Grothendieck residue. Still, we will show that the integral can be expressed through power sums of the reciprocal roots of the system $f(z)=0$. 
Using the geometric series we expand the function $1 / f_{1} \cdot \ldots \cdot f_{n}$ on $\Gamma_{P}$. More precisely,

$$
\begin{aligned}
\frac{1}{f_{1} \cdot \ldots \cdot f_{n}} & =\frac{1}{z^{m_{1}+\ldots+m_{n}} P_{1} \cdot \ldots \cdot P_{n}\left(1+\frac{Q_{1}}{z^{m_{1} P_{1}}}\right) \cdot \ldots \cdot\left(1+\frac{Q_{n}}{z^{m_{n}} P_{n}}\right)} \\
& =\frac{1}{z^{m_{1}+\ldots+m_{n}} P_{1} \cdot \ldots \cdot P_{n}} \sum_{\alpha \in \mathbb{Z}_{+}^{n}}\left(-\frac{Q_{1}}{z^{m_{1}} P_{1}}\right)^{\alpha_{1}} \cdot \ldots \cdot\left(-\frac{Q_{n}}{z^{m_{n}} P_{n}}\right)^{\alpha_{n}} \\
& =\sum_{\alpha \in \mathbb{Z}_{+}^{n}}(-1)^{|\alpha|} \frac{Q^{\alpha}}{z^{\left(\alpha_{1}+1\right) m_{1}+\ldots+\left(\alpha_{n}+1\right) m_{n} P^{\alpha+I}}}
\end{aligned}
$$

where $\alpha+I=\left(\alpha_{1}+1, \ldots, \alpha_{n}+1\right)$. Since the series converges uniformly on $\Gamma_{P}$, termwise integration yields

$$
\mathcal{I}(s+I)=\sum_{\alpha \in \mathbb{Z}_{+}^{n}} \frac{(-1)^{|\alpha|}}{(2 \pi \imath)^{n}} \int_{\Gamma_{P}} \frac{1}{z^{s+I}} \frac{Q^{\alpha} J_{f} d z}{z^{\left(\alpha_{1}+1\right) m_{1}+\ldots+\left(\alpha_{n}+1\right) m_{n} P^{\alpha+I}}}
$$

where $J_{f}$ is the Jacobian of system (2.1).

Our next objective is to show that only finitely many summands in (3.1) are different from zero. Since the cycle $\Gamma_{P}$ is a circular set, the only non-zero integrals may be those whose numerator (counted with $d z$ ) has the same degree of homogeneity as that of the denominator. The degree of the denominator of any term in (3.1) is equal to

$$
|s|+n+\left(\alpha_{1}+1\right)\left|m_{1}\right|+\ldots+\left(\alpha_{n}+1\right)\left|m_{n}\right|+\left(\alpha_{1}+1\right) k_{1}+\ldots+\left(\alpha_{n}+1\right) k_{n}
$$

while the degree of the numerator is at least

$$
\left|m_{1}\right|+k_{1}+\ldots+\left|m_{n}\right|+k_{n}-n+\alpha_{1}\left(\left|m_{1}\right|+k_{1}+1\right)+\ldots+\alpha_{n}\left(\left|m_{n}\right|+k_{n}+1\right)+n .
$$

Hence it follows that the number of nonzero summands in (3.1) is at most $|s|+n$, for the degree of the numerator is at least $|\alpha|-|s|-n$ greater than the degree of the denominator.

Summarising we arrive at the following result.

Lemma 3.1. If defined as above, $\mathcal{I}(s+I)$ just amounts to

$$
\mathcal{I}(s+I)=\sum_{|\alpha| \leq|s|+n} \frac{(-1)^{|\alpha|}}{(2 \pi \imath)^{n}} \int_{\Gamma_{P}} \frac{1}{z^{s+I}} \frac{Q^{\alpha} J_{f} d z}{z^{\left(\alpha_{1}+1\right) m_{1}+\ldots+\left(\alpha_{n}+1\right) m_{n} P^{\alpha+I}}} .
$$

\section{TRANSFORMATION UNDER AN INVOLUtion}

Consider the transformation $z_{j}=1 / w_{j}, j=1, \ldots, n$. This is a biholomorphic involution $(\mathbb{C} \backslash\{0\})^{n} \rightarrow(\mathbb{C} \backslash\{0\})^{n}$.

We get

$$
\begin{aligned}
f_{j}\left(\frac{1}{w}\right) & =\frac{1}{w^{m_{j}}} P_{j}\left(\frac{1}{w}\right)+Q_{j}\left(\frac{1}{w}\right) \\
& =\frac{1}{w^{M_{j}}}\left(w^{M_{j}-m_{j}} P_{j}\left(\frac{1}{w}\right)+w^{M_{j}} Q_{j}\left(\frac{1}{w}\right)\right)
\end{aligned}
$$


where $M_{j}=\left(M_{j, 1}, \ldots, M_{j, n}\right)$ is a multi-index of $\mathbb{Z}^{n}$ to be chosen. To this end, we introduce

$$
\begin{aligned}
& \tilde{P}_{j}(w)=w^{M_{j}-m_{j}} P_{j}\left(\frac{1}{w}\right) \text { and } \\
& \tilde{Q}_{j}(w)=w^{M_{j}} Q_{j}\left(\frac{1}{w}\right) .
\end{aligned}
$$

We require both $\tilde{P}_{j}$ and $\tilde{Q}_{j}$ to be polynomials. The degree of each monomial of $\tilde{P}_{j}$ and $\tilde{Q}_{j}$ in $w_{k}$ is at least

$$
\begin{aligned}
& M_{j, k}-m_{j, k}-\operatorname{deg}_{z_{k}} P_{j}, \\
& M_{j, k}-\operatorname{deg}_{z_{k}} Q_{j},
\end{aligned}
$$

respectively. This suggests the choice

$$
M_{j, k}:=\max \left\{m_{j, k}+\operatorname{deg}_{z_{k}} P_{j}, \operatorname{deg}_{z_{k}} Q_{j}\right\}
$$

for any $j, k=1, \ldots, n$.

Under (4.1), every $\tilde{f}_{j}=\tilde{P}_{j}+\tilde{Q}_{j}$ is a polynomial and $\tilde{f}_{j}(w)=w^{M_{j}} f_{j}(1 / w)$, for $j=1, \ldots, n$. Since $\operatorname{deg} \tilde{P}_{j}=\left|M_{j}\right|-\left|m_{j}\right|-k_{j}$ and $\operatorname{deg} \tilde{Q}_{j}=\left|M_{j}\right|-\operatorname{deg} Q_{j}$, we conclude that $\operatorname{deg} \tilde{P}_{j}>\operatorname{deg} \tilde{Q}_{j}$.

In what follows we assume that the system

$$
\left\{\begin{array}{lll}
\tilde{P}_{1}(w) & =0 \\
\tilde{P}_{n}(w) & \cdots & 0
\end{array}\right.
$$

has the only common zero at the origin $w=0$. This implies that the algebraic system $\tilde{f}(w)=0$ meets the condition of Bézout's theorem.

By assumption, the cycle $\Gamma_{P}$ lies in $(\mathbb{C} \backslash\{0\})^{n}$. Under the involution $z=1 / w$ it transforms to a compact cycle of the form

$$
\tilde{\Gamma}_{P}=\left\{w \in \mathbb{C}^{n}:\left|\tilde{P}_{j}(w)\right|=\left|w^{M_{j}-m_{j}}\right| \varepsilon_{j} \text { for } j=1, \ldots, n\right\} .
$$

Our next goal is to transform the integral $\mathcal{I}(s+I)$ under the change of variables $z=1 / w$. By the above,

$$
\begin{aligned}
\frac{d f_{j}\left(\frac{1}{w}\right)}{f_{j}\left(\frac{1}{w}\right)} & =\frac{d\left(\frac{1}{w^{M_{j}}} \tilde{f}_{j}(w)\right)}{\frac{1}{w^{M_{j}}} \tilde{f}_{j}(w)} \\
& =\frac{d \frac{1}{w^{M_{j}}}}{\frac{1}{w^{M_{j}}}}+\frac{d \tilde{f}_{j}(w)}{\tilde{f}_{j}(w)} \\
& =\frac{d \tilde{f}_{j}(w)}{\tilde{f}_{j}(w)}-\sum_{k=1}^{n} M_{j, k} \frac{d w_{k}}{w_{k}}
\end{aligned}
$$

whence $\mathcal{I}(s+I)$ transforms to

$$
\frac{(-1)^{n}}{(2 \pi \imath)^{n}} \int_{\tilde{\Gamma}_{P}} w^{s+I}\left(\frac{d \tilde{f}_{1}(w)}{\tilde{f}_{1}(w)}-\sum_{k=1}^{n} M_{1, k} \frac{d w_{k}}{w_{k}}\right) \wedge \ldots \wedge\left(\frac{d \tilde{f}_{n}(w)}{\tilde{f}_{n}(w)}-\sum_{k=1}^{n} M_{n, k} \frac{d w_{k}}{w_{k}}\right),
$$

the factor $(-1)^{n}$ being due to the change of orientation. 
The analytic polyhedron

$$
\Pi=\left\{w \in \mathbb{C}^{n}:\left|\tilde{P}_{j}(w)\right|>\left|w^{M_{j}-m_{j}}\right| \varepsilon_{j} \text { for } j=1, \ldots, n\right\}
$$

lies in the product $(\mathbb{C} \backslash\{0\})^{n}$. Moreover, it agrees with the family of divisors $D_{j}:=\left\{w \in \mathbb{C}^{n}: \tilde{P}_{j}(w)=0\right\}$ in the sense that the $j$-th face of $b \Pi$ does not intersect $D_{j}$, for each $j=1, \ldots, n$. Since $(\mathbb{C} \backslash\{0\})^{n}$ is a domain of holomorphy, we may apply the principle of separating cycles, cf. [Tsi92, §9], which immediately implies

$\mathcal{I}(s+I)=\frac{(-1)^{n}}{(2 \pi \imath)^{n}} \int_{\Gamma_{\tilde{P}}} w^{s+I}\left(\frac{d \tilde{f}_{1}(w)}{\tilde{f}_{1}(w)}-\sum_{k=1}^{n} M_{1, k} \frac{d w_{k}}{w_{k}}\right) \wedge \ldots \wedge\left(\frac{d \tilde{f}_{n}(w)}{\tilde{f}_{n}(w)}-\sum_{k=1}^{n} M_{n, k} \frac{d w_{k}}{w_{k}}\right)$.

The integral on the right-hand side is a linear combination of integrals of the form

$$
\int_{\Gamma_{\tilde{P}}} w^{s+I}\left(\frac{d \tilde{f}_{j_{1}}(w)}{\tilde{f}_{j_{1}}(w)} \wedge \ldots \wedge \frac{d \tilde{f}_{j_{k}}(w)}{\tilde{f}_{j_{k}}(w)}\right) \wedge\left(\frac{d w_{j_{k+1}}}{w_{j_{k+1}}} \wedge \ldots \wedge \frac{d w_{j_{n}}}{w_{j_{n}}}\right)
$$

where $1 \leq j_{1}<\ldots<j_{k} \leq n$ and $1 \leq j_{k+1}<\ldots<j_{n} \leq n$. These integrals vanish unless $k=n$. Indeed, on the cycle $\Gamma_{\tilde{P}}$ the inequality $\left|\tilde{P}_{j}(w)\right|>\left|\tilde{Q}_{j}(w)\right|$ is fulfilled for any $j=1, \ldots, n$. Hence, expanding the integrand of (4.2) as a geometric series on $\Gamma_{\tilde{P}}$ just as in Section 3 we deduce that (4.2) is actually the sum of integrals of the form

$$
\int_{\Gamma_{\tilde{P}}} w^{s+I} h_{J, \alpha}(w)\left(\frac{d w_{j_{1}}}{\tilde{P}_{j_{1}}^{\alpha_{1}}} \wedge \ldots \wedge \frac{d w_{j_{k}}}{\tilde{P}_{j_{k}}^{\alpha_{k}}}\right) \wedge\left(\frac{d w_{j_{k+1}}}{w_{j_{k+1}}} \wedge \ldots \wedge \frac{d w_{j_{n}}}{w_{j_{n}}}\right)
$$

with $h_{J, \alpha}$ a polynomial. Since the cycle $\Gamma_{\tilde{P}}$ is the skeleton of the bounded analytic polyhedron $\left\{w \in \mathbb{C}^{n}:\left|\tilde{P}_{j}(w)\right|<\varepsilon_{j}\right.$ for $\left.j=1, \ldots, n\right\}$, it follows that $\Gamma_{\tilde{P}}=b F_{j_{k+1}}$, where

$$
F_{j_{k+1}}=\left\{w \in \mathbb{C}^{n}:\left|\tilde{P}_{j}(w)\right|=\varepsilon_{j} \text { for } j \neq j_{k+1},\left|\tilde{P}_{j_{k+1}}(w)\right|<\varepsilon_{j_{k+1}}\right\} .
$$

The integrand of (4.3) has no singularities on the face $F_{j_{k+1}}$, which enables us to apply the Stokes formula to (4.3). We conclude this way that all integrals (4.3) vanish, and so do the integrals (4.2) for $k=1, \ldots, n-1$, as desired. We have proved the following formula.

Lemma 4.1. If $Q_{j}$ are polynomials satisfying (4.1), then

$$
\mathcal{I}(s+I)=\frac{(-1)^{n}}{(2 \pi \imath)^{n}} \int_{\Gamma_{\tilde{P}}} w^{s+I} \frac{d \tilde{f}_{1}(w)}{\tilde{f}_{1}(w)} \wedge \ldots \wedge \frac{d \tilde{f}_{n}(w)}{\tilde{f}_{n}(w)} .
$$

\section{POWER SUMS}

By the theorem of Bézout, cf. for instance [Tsi92, § 19], the system

$$
\left\{\begin{array}{lll}
\tilde{f}_{1}(w) & =0 \\
\tilde{f}_{n}(w) & \cdots & 0
\end{array}\right.
$$

has a finite number of roots in $\mathbb{C}^{n}$, the roots being counted with their multiplicities. This number is actually equal to the product of the degrees of the polynomials $\tilde{P}_{j}(w)$.

Denote by $b_{k}=\left(b_{k, 1}, \ldots, b_{k, n}\right)$ the roots of (5.1) which lie away from the coordinate hyperplanes in $\mathbb{C}^{n}$. Here, the index $k$ runs over the set $1, \ldots, N$, where 
$N$ is the number of roots. Setting $a_{k, j}=1 / b_{k, j}$, we easily see that the points $a_{k}=\left(a_{k, 1}, \ldots, a_{k, n}\right)$ form the set of all zeros of (2.1) lying away from the coordinate hyperplanes.

Write

$$
\begin{aligned}
\sigma(s+I) & =\sum_{k=1}^{N} a_{k}^{-s-I} \\
& =\sum_{k=1}^{N} b_{k}^{s+I}
\end{aligned}
$$

for a power sum of the zeros of system (2.1) which lie in the complement of the coordinate hyperplanes in $\mathbb{C}^{n}$.

Theorem 5.1. For any system (2.1) with polynomials $P_{j}$ and $Q_{j}$ satisfying (4.1), the equality holds

$$
\mathcal{I}(s+I)=(-1)^{n} \sigma(s+I) .
$$

Proof. By Lemma 4.1, we get

$$
\mathcal{I}(s+I)=\frac{(-1)^{n}}{(2 \pi \imath)^{n}} \int_{\Gamma_{\tilde{P}}} w^{s+I} \frac{d \tilde{f}}{\tilde{f}} .
$$

We now invoke the Rouché principle for residues, cf. Theorem 4.8 of [AYu83] or $[$ Tsi92, § 8]. It gives

$$
\begin{aligned}
\mathcal{I}(s+I) & =\frac{(-1)^{n}}{(2 \pi \imath)^{n}} \int_{\Gamma_{\tilde{f}}} w^{s+I} \frac{d \tilde{f}}{\tilde{f}} \\
& =(-1)^{n} \sum_{k=1}^{N} b_{k}^{s+I}
\end{aligned}
$$

because on $\Gamma_{\tilde{P}}$ the inequalities $\left|\tilde{P}_{j}(w)\right|>\left|\tilde{Q}_{j}(w)\right|$ are fulfilled, for $j=1, \ldots, n$. This establishes the formula.

\section{Generalisation to Meromorphic maps}

We now consider more general systems (2.1). Suppose every $f_{j}, j=1, \ldots, n$, has the form

$$
f_{j}(z)=\frac{f_{j}^{(1)}(z)}{f_{j}^{(2)}(z)}
$$

where $f_{j}^{(1)}(z)$ and $f_{j}^{(2)}(z)$ are entire functions on $\mathbb{C}^{n}$ with $f_{j}^{(2)}(0) \neq 0$. We moreover require both the numerators and the denominators to admit infinite product expansions

$$
\begin{aligned}
f_{j}^{(1)}(z) & =\prod_{k=1}^{\infty} f_{j, k}^{(1)}(z), \\
f_{j}^{(2)}(z) & =\prod_{k=1}^{\infty} f_{j, k}^{(2)}(z)
\end{aligned}
$$

which converge absolutely and uniformly on compact subsets of $\mathbb{C}^{n}$, each factor being of the form (2.1) with polynomials $P_{j}$ and $Q_{j}$ satisfying condition (4.1). 
For any multi-index $\left(k_{1}, \ldots, k_{n}\right) \in \mathbb{N}^{n}$ and any $n$-tuple $\left(i_{1}, \ldots, i_{n}\right)$ of numbers 1 and 2 , the system of algebraic equations

$$
\left\{\begin{array}{c}
f_{1, k_{1}}^{\left(i_{1}\right)}(z)=0 \\
f_{n, k_{n}}^{\left(i_{n}\right)}(z)=0
\end{array}\right.
$$

has at most a finite number of roots away from the coordinate hyperplanes in $\mathbb{C}^{n}$. The roots of all systems (6.2) which lie in the complement of the coordinate hyperplanes form at most a countable discrete set in $\mathbb{C}^{n}$. Let $a_{1}, a_{2}, \ldots$ be a numbering of the roots which are counted with their multiplicities. Our basic assumption is that the series

$$
\sum_{k=1}^{\infty} \frac{1}{\left|a_{k}^{I}\right|}
$$

converges.

Given any multi-index $s \in \mathbb{Z}_{+}^{n}$, we are interested in the power sums of the form

$$
\sigma(s+I)=\sum_{k=1}^{\infty}(-1)^{p(k)} a_{k}^{-s-I}
$$

where $p(k)$ is the number of functions $f_{j, k_{j}}^{(2)}$ in the system (6.2) defining $a_{k}$. The series on the right-hand side converges, which is due to (6.3). For the system (6.1), the points $a_{k}$ are either zeros or poles.

Theorem 6.1. Under the above assumptions on (6.1), it follows that

$$
\mathcal{I}(s+I)=(-1)^{n} \sigma(s+I) .
$$

Proof. A trivial verification shows that

$$
\frac{d f_{j}}{f_{j}}=\frac{d f_{j}^{(1)}}{f_{j}^{(1)}}-\frac{d f_{j}^{(2)}}{f_{j}^{(2)}},
$$

hence

$$
\begin{aligned}
\frac{d f}{f} & =\left(\frac{d f_{1}^{(1)}}{f_{1}^{(1)}}-\frac{d f_{1}^{(2)}}{f_{1}^{(2)}}\right) \wedge \ldots \wedge\left(\frac{d f_{n}^{(1)}}{f_{n}^{(1)}}-\frac{d f_{n}^{(2)}}{f_{n}^{(2)}}\right) \\
& =\sum(-1)^{o(i)} \frac{d f_{1}^{\left(i_{1}\right)}}{f_{1}^{\left(i_{1}\right)}} \wedge \ldots \wedge \frac{d f_{n}^{\left(i_{n}\right)}}{f_{n}^{\left(i_{n}\right)}}
\end{aligned}
$$

where the sum is over all possible $n$-tuples $i=\left(i_{1}, \ldots, i_{n}\right)$ of numbers 1 and 2 , and $o(i)$ is the number of entries 2 in $i$.

We are thus reduced to proving the theorem for entire functions $f_{j}=f_{j}^{(1)}$. In this case we have

$$
\begin{aligned}
\frac{d f}{f} & =\frac{d \prod_{k=1}^{\infty} f_{1, k}}{\prod_{k=1}^{\infty} f_{1, k}} \wedge \ldots \wedge \frac{d \prod_{k=1}^{\infty} f_{n, k}}{\prod_{k=1}^{\infty} f_{n, k}} \\
& =\sum_{k_{1}, \ldots, k_{n}=1}^{\infty} \frac{d f_{1, k_{1}}}{f_{1, k_{1}}} \wedge \ldots \wedge \frac{d f_{n, k_{n}}}{f_{n, k_{n}}} .
\end{aligned}
$$


Hence it follows that the integral $\mathcal{I}(s+I)$ is equal to the sum of integrals of the form

$$
\frac{1}{(2 \pi \imath)^{n}} \int_{\Gamma_{P}} \frac{1}{z^{s+I}} \frac{d f_{1, k_{1}}}{f_{1, k_{1}}} \wedge \ldots \wedge \frac{d f_{n, k_{n}}}{f_{n, k_{n}}} .
$$

Since for such integrals the desired formula is established by Theorem 5.1, the assertion follows.

\section{EXPLICIT EVALUATION}

In this section we show that the integral $\mathcal{I}(s+I)$ can be evaluated through Taylor coefficients of the functions $f_{1}, \ldots, f_{n}$. Since the system $\tilde{P}(w)=0$ has the only root at the origin $0 \in \mathbb{C}^{n}$, the theorem of Hilbert on roots (cf. for instance [Tsi92, $\S 20]$ ) guarantees the existence of numbers $N_{1}, \ldots, N_{n}$ and a matrix of homogeneous polynomials

$$
\tilde{A}=\left(\tilde{a}_{i, j}(w)\right) \begin{gathered}
i=1, \ldots, n \\
j=1, \ldots, n
\end{gathered}
$$

such that $\operatorname{deg} \tilde{a}_{i, j}=N_{i}-\operatorname{deg} \tilde{P}_{j}$ and

$$
w_{i}^{N_{i}}=\sum_{j=1}^{n} \tilde{a}_{i, j}(w) \tilde{P}_{j}(w)
$$

for $i=1, \ldots, n$. Substituting $w=1 / z$ into this equality and multiplying both its sides by the lowest common multiple, we get

$$
z^{\delta_{i}}=\sum_{j=1}^{n} a_{i, j}(z) P_{j}(z)
$$

for $i=1, \ldots, n$, with some multi-indices $\delta_{i} \in \mathbb{N}^{n}$ and polynomials $a_{i, j}(z)$.

For a multi-index $\alpha \in \mathbb{Z}_{+}^{n \times n}$ with entries $\alpha_{i, j}$, we set

$$
\alpha_{i, \cdot}=\left(\alpha_{i, 1}, \ldots, \alpha_{i, n}\right), \quad \alpha_{\cdot, j}=\left(\begin{array}{c}
\alpha_{1, j} \\
\vdots \\
\alpha_{n, j}
\end{array}\right)
$$

and $|\alpha|=\sum_{i, j=1}^{n} \alpha_{i, j}$.

Theorem 7.1. Let $f_{1}, \ldots, f_{n}$ fulfil the hypotheses of Theorem 5.1 or Theorem 6.1. Then,

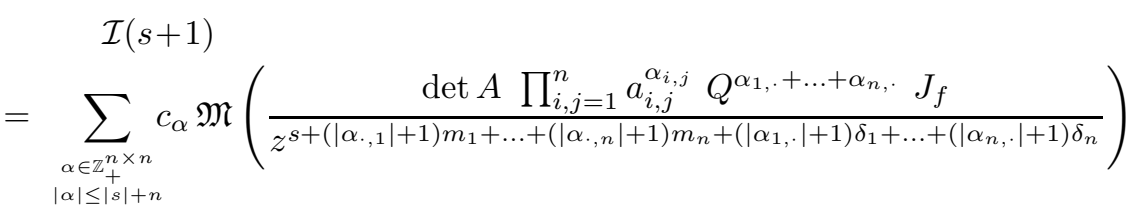

where

$$
c_{\alpha}=(-1)^{|\alpha|} \frac{\prod_{i=1}^{n}\left|\alpha_{i, \cdot}\right| !}{\prod_{i, j=1}^{n} \alpha_{i, j} !}
$$

and $\mathfrak{M}$ is the functional assigning the constant term to a Laurent polynomial. 
Proof. We first consider a system $f_{1}, \ldots, f_{n}$ satisfying the conditions of Theorem 5.1. In this case $Q_{1}, \ldots, Q_{n}$ are polynomials. Changing the variables in (7.1) by $z_{j}=1 / w_{j}$, for $j=1, \ldots, n$, we obtain

$$
\begin{aligned}
\frac{1}{w^{\delta_{i}}} & =\sum_{j=1}^{n} a_{i, j}\left(\frac{1}{w}\right) P_{j}\left(\frac{1}{w}\right) \\
& =\sum_{j=1}^{n} a_{i, j}\left(\frac{1}{w}\right) \frac{1}{w^{M_{j}-m_{j}}} \tilde{P}_{j}(w)
\end{aligned}
$$

where the multi-indices $M_{1}, \ldots, M_{n}$ are defined in Theorem 5.1.

Multiplying these equalities by the lowest common multiples leads to a system of polynomials. In fact, there are multi-indices $I_{1}, \ldots, I_{n}$ of $\mathbb{Z}_{+}^{n}$ with the property that

$$
w^{I_{i}-\delta_{i}}=\sum_{j=1}^{n} a_{i, j}\left(\frac{1}{w}\right) w^{I_{i}-M_{j}+m_{j}} \tilde{P}_{j}(w)
$$

for $i=1, \ldots, n$, where

$$
w^{I_{i}-M_{j}+m_{j}} a_{i, j}\left(\frac{1}{w}\right)=\tilde{a}_{i, j}(w)
$$

are polynomials of degree $N_{i}-\operatorname{deg} \tilde{P}_{j}$ and $I_{i}-\delta_{i}=N_{i} e_{i}$. As usual, we denote by $e_{i} \in \mathbb{Z}_{+}^{n}$ the multi-index whose entries are zero but 1 at position $i$. We thus arrive at the equalities

$$
w_{i}^{N_{i}}=\sum_{j=1}^{n} \tilde{a}_{i, j}(w) \tilde{P}_{j}(w)
$$

we have started with.

In the proof of Theorem 5.1 we have got the formula

$$
\mathcal{I}(s+I)=\frac{(-1)^{n}}{(2 \pi \imath)^{n}} \int_{\Gamma_{\tilde{P}}} w^{s+I} \frac{d \tilde{f}}{\tilde{f}} .
$$

For such integrals, Theorem 8.1 of [BKL98] gives, under condition (7.4), the explicit formula

(7.5) $\mathcal{I}(s+1)=(-1)^{n} \sum_{\substack{\alpha \in \mathbb{Z}_{+}^{n \times n} \\|\alpha| \leq|s|+n}} c_{\alpha} \mathfrak{M}\left(\frac{w^{s+I} \operatorname{det} \tilde{A} \prod_{i, j=1}^{n} \tilde{a}_{i, j}^{\alpha_{i, j}} \tilde{Q}^{\alpha_{1, \cdot}+\ldots+\alpha_{n, \cdot}} J_{\tilde{f}}}{\prod_{j=1}^{n} w_{j}^{\left(\left|\alpha_{j,},\right|+1\right) N_{j}-1}}\right)$,

$J_{\tilde{f}}$ being the Jacobian of the system $\tilde{f}_{j}=\tilde{P}_{j}+\tilde{Q}_{j}$, where $j=1, \ldots, n$.

To obtain a formula in terms of the Taylor coefficients of the genuine system, we change the variables by $w_{j}=1 / z_{j}$, for $j=1, \ldots, n$. Analysis similar to that in the proof of Theorem 5.1 shows that

$$
\begin{aligned}
& \tilde{P}_{j}\left(\frac{1}{z}\right)=z^{m_{j}-M_{j}} P_{j}(z), \\
& \tilde{Q}_{j}\left(\frac{1}{z}\right)=z^{-M_{j}} Q_{j}(z)
\end{aligned}
$$

and $\tilde{f}_{j}(1 / z)=z^{-M_{j}} f_{j}(z)$. Hence

$$
\tilde{Q}^{\alpha_{1, \cdot}+\ldots+\alpha_{n, \cdot}}\left(\frac{1}{z}\right)=\frac{Q^{\alpha_{1, \cdot}+\ldots+\alpha_{n, \cdot}}(z)}{z^{\left|\alpha_{\cdot, 1}\right| M_{1}+\ldots+\left|\alpha_{\cdot, n}\right| M_{n}}},
$$


and the entries of the Jacobi matrix of $\tilde{f}$ are

$$
\begin{aligned}
\frac{\partial \tilde{f}_{j}}{\partial w_{k}} & =\frac{\partial \tilde{f}_{j}(1 / z)}{\partial\left(1 / z_{k}\right)} \\
& =-z_{k}^{2}\left(\frac{1}{z^{M_{j}}} \frac{\partial f_{j}}{\partial z_{k}}-\frac{M_{j, k}}{z^{M_{j}+e_{k}}} f_{j}\right) \\
& =\frac{z_{k}}{z^{M_{j}}}\left(-z_{k} \frac{\partial f_{j}}{\partial z_{k}}+M_{j, k} f_{j}\right)
\end{aligned}
$$

for $j, k=1, \ldots, n$. The Jacobian of $\tilde{f}$ is therefore of the form

$$
J_{\tilde{f}}=(-1)^{n} \frac{z_{1}^{2} \cdot \ldots \cdot z_{n}^{2}}{z^{M_{1}+\ldots+M_{n}}} J_{f}+\sum_{j=1}^{n} c_{j} f_{j},
$$

where $c_{j}(z)$ are rational functions with denominators being monomials. When substituted into (7.5), the second term of (7.6) gives zero contribution since the integral $\mathcal{I}(s+I)$ just amounts to the sum $(-1)^{n} \sigma(s+I)$ which is evaluated at the roots of $f=0$.

Further, an easy computation computation shows that

$$
\operatorname{det} \tilde{A}\left(\frac{1}{z}\right)=\frac{\operatorname{det} A(z)}{z^{I_{1}+\ldots+I_{n}-M_{1}-\ldots-M_{n}+m_{1}+\ldots+m_{n}}},
$$

and so after substituting all the expressions into (7.5) we obtain formula (7.2), as desired.

In the case when $Q_{1}, \ldots, Q_{n}$ fail to be polynomials we apply (7.5) to partial sums of the Taylor series of these functions. Since the functional $\mathfrak{M}$ vanishes on summands whose numerators are of sufficiently large degree, the equality (7.2) follows.

Acknowledgements This article was written during the stay of first two authors at the Institute of Mathematics, University of Potsdam. They gratefully acknowledge the financial support of the Deutsche Forschungsgemeinschaft and the RFFI grant 02-01-00167. 


\section{REFERENCES}

[AYu83] L. A. Aizenberg and A. P. Yuzhakov, Integral Representations and Residues in Multidimensional Complex Analysis, Transl. Math. Monographs, AMS, Providence, RI, 1983.

[BKL98] V. Bykov, A. Kytmanov, and M. Lazman, Elimination Methods in Polynomial Computer Algebra, Kluwer Academic Publishers, Dordrecht, 1998.

[BKO96] V. I. Bykov, A. M. Kytmanov, and T. A. Osetrova, Computer algebra of polynomials. A modified elimination method, Dokl. of RAN 350 (1996), no. 4, 443-445.

[GH78] P. A. Griffiths, and J. Harris, Principles of Algebraic Geometry, John Wiley and Sons, New York, 1978.

[KPO5] A. M. Kytmanov and Z. E. Potapova, Formulas for finding power sums of the roots of systems of meromorphic functions, Izv. VUZ. Mat. ?? (2005), 16 pp.

[Tsi92] A. K. TsikH, Multidimensional Residues and Their Applications, Transl. Math. Monographs, AMS, Providence, RI, 1992.

(Alexander Kytmanov) Krasnoyarsk State University, Pr. Svobodnyi 79, 660041 KrasNOYARSK, RUSSIA

E-mail address: kytmanov@lan.krasu.ru

(Simona Myslivets) Krasnoyarsk State University, Pr. Svobodnyi 79, 660041 KrasnoYARSK, RUSSIA

E-mail address: simona@lan.krasu.ru

(Nikolai Tarkhanov) Universität Potsdam, Institut für Mathematik, Postfach 601553 , 14415 Potsdam, Germany

E-mail address: tarkhanov@math.uni-potsdam.de 